\title{
Medicina de Emergência na FMRP-USP - Você pode fugir, mas não há como se esconder!
}

\author{
Emergency Medicine in the Medical School of Ribeirão Preto \\ - University of São Paulo - You can run, but you can't hide!
}

\author{
Antonio Pazin Filho, Sandro Scarpelini
}

\begin{abstract}
RESUMO
A Medicina de Emergência é uma especialidade médica que objetiva o processo de atendimento ágil e eficaz em situações de agravo agudo à saúde das pessoas, embora ainda não seja considerada como tal no Brasil. Está extremamente relacionada com outras áreas de atuação e deve garantir um fluxo ("cascata") de atendimento tanto no aspecto estrutural (pré, intra e pós-hospitalar) como no aspecto inter-disciplinar. Várias iniciativas tem buscado inseri-la na realidade brasileira, sendo que o foco atual está centrado nas sociedades de especilidades médicas pela própria demanda de mercado. Em que pese a necessidade desta organização no curto prazo, a formação de profissionais capacitados em emergência deve se iniciar na própria graduação, sendo esta a única iniciativa que irá garantir a modificação cultural necessária no médio e no longo prazos. A Faculdade de Medicina de Ribeirão Preto da Universidade de São Paulo (FMRP-USP) já realizou diversas tentativas para esta inserção curricular ao longo de sua história, algumas delas resgatadas neste artigo. Atualmente há um novo projeto que propõe sua inserção em um eixo longitudinal que busca respeitar as necessidades da especialidade, está embasado na infraestrutura disponível para locais de ensino e se adapta ao modelo educativo proposto pela Comissão de Graduação. A proposta é sumarizada, apontando seus princípios e metas para cada etapa e reforçando a necessidade de apoio e fiscalização institucionais para seu sucesso.
\end{abstract}

Palavras-chave: Medicina de Emergência. Educação Médica. História da Medicina.

"Aqueles que não se recordam do passado estão condenados a repeti-lo" (George Santayana)

Em que pese a inexistência de uma especialidade médica regulamentada em Emergência no contexto brasileiro, é inegável sua importância na prática diária da medicina. A experiência adquirida por países desenvolvidos demonstra que, à medida que o sistema de saúde se organiza, a Medicina de Emergência se estrutura como especialidade médica, forçando as instituições universitárias a desenvolver mecanismos para propiciar a formação de profissionais especializados na área. ${ }^{1}$

\footnotetext{
${ }^{1}$ Docente da Divisão de Emergências Clínicas do Departamento de Clínica Médica da Faculdade de Medicina de Ribeirão Preto da Universidade de São Paulo; Coordenador da Unidade de Emergência do Hospital das Clínicas da Faculdade de Medicina de Ribeirão Preto da Universidade de São Paulo.

${ }^{2}$ Docente da Disciplina de Cirurgia de Urgência e Trauma do Departamento de Cirurgia e Anatomia da Faculdade de Medicina de Ribeirão Preto da Universidade de São Paulo; Ex-Diretor Médico da Unidade de Emergência do HCFMRP, Ex- Coordenado do SAMU de Ribeirão Preto; Diretor Executivo da Fundação de Apoio ao Ensino, Pesquisa e Assistência do HCFMRP-USP.
}

Correspondência: Prof. Dr. Antonio Pazin-Filho Unidade de Emergência do HCFMRP-USP R. Bernardino de Campus, 1000 14010-030 - Ribeirão Preto - SP Telefone - 16-3610-1110 apazin@fmrp.usp.br

Artigo recebido em 1\%07/2010 Aprovado em 30/08/2010 
Outra constatação da experiência adquirida por estes países é decorrente do perfil epidemiológico dos pacientes que buscam o atendimento de emergência e sua evolução histórica. Este perfil epidemiológico tem se alterado nas últimas décadas, sendo que os atendimentos emergenciais decorrentes de acidentes automobilísticos estão deixando de ser mais prevalentes em comparação com as emergências clínicas, provavelmente em decorrência da eficácia de legislação preventiva e da mudança do perfil populacional, nos países desenvolvidos. ${ }^{2}$ Os hábitos e condições de vida, bem como o envelhecimento da população, que acompanham o desenvolvimento implicam em aumento da incidência de doenças crônicas. Paralelamente a este processo, novos tratamentos e melhora das condições de acompanhamento destas condições crônicas tem aumentado sua prevalência e a apresentação nas salas de emergência traduz estas mudanças de modo bem acurado. ${ }^{3}$ Situações anteriormente raras nas salas de emergência, como o atendimento a pacientes oncológicos, se tornam mais frequentes à medida que se implantam as melhorias do sistema de saúde e os benefícios do desenvolvimento da medicina. Grande parte deste contexto descrito tem sido vivenciado pelo Brasil, em particular pela Divisão Regional de Saúde de Ribeirão Preto do Estado de São Paulo - DRS XIII.

A própria Organização Mundial de Saúde (OMS) reconheceu recentemente a necessidade de se incluir emergência como uma ação prioritária nas suas iniciativas, pois à medida que o sistema de saúde se organiza o atendimento para emergência se impõe. ${ }^{4}$ A experiência desta Organização demonstra que em países em desenvolvimento com nível elevado de pobreza, o estabelecimento de ações de caráter preventivo, sem que se ofereça atendimento para os agravos agudos de saúde, diminui a confiança no sistema e coloca em risco toda a intervenção pretendida. ${ }^{4,6}$

A caracterização da Emergência como área de especialização médica enfrenta grande refratariedade no meio profissional, por preconceito e defesa de um nicho de mercado, muito embora seja nítido o paralelismo com o processo de criação de outras áreas. ${ }^{2 ; 7 ; 8}$ Mais do que tudo, ainda impera o conceito de que cada especialidade possui suas condições clínicas de atendimento de emergência, que lhe são peculiares e não podem ser manejadas por um médico "não-especialista". Quando confrontados com a realidade da inexistência de um profissional de cada especialidade médica disponível nas salas de emergência do país, passa-se a propor diretrizes de atendimento. No en- tanto, estas diretrizes são desenvolvidas por profissionais que não atuam na sala de emergência e, portanto, desconhecem a realidade deste ambiente.

Mais preocupante do que isto é a omissão da academia na abordagem sistemática do tema, transferindo para as sociedades médicas a responsabilidade da definição e da criação da especialidade Medicina de Emergência. No momento, a Emergência Médica é considerada como área de atuação em Clínica Médica, e assim cadastrada na Comissão Nacional de Residência Médica e na Associação Médica Brasileira. ${ }^{9}$ Isto tem ocasionado divergência de opiniões e o surgimento de duas sociedades médicas - Associação Brasileira de Medicina de Urgência e Emergência (ABRAMURGEM - http://www.abramurgem.org.br/ portal/) e Associação Brasileira de Medicina de Emergência (ABRAMEDE - http://www.abramede.com.br/ tag/medicina-de-emergencia/). É lógico que o estabelecimento da Medicina de Emergência como especialidade tem implicações muito maiores, pois a consolidação de uma especialidade deverá passar necessariamente por um plano de carreira, delimitação de locais de trabalho, treinamento continuado e defesa profissional. Caso isto não ocorra, mesmo que se estabeleça a especialidade, poderá haver escassez de profissionais no mercado de trabalho, a exemplo do que já se vivencia nos Estados Unidos e no Brasil em relação à profissionais para a Terapia Intensiva e Saúde da Família. 9;10 Para tanto as associações médicas têm exercido papel importante em nossa sociedade, mas a unificação em torno de um objetivo comum é premente.

Do ponto de vista acadêmico, a discussão sobre a Medicina de Emergência também é controversa, incluindo itens de linhas de fomento à pesquisa $\mathrm{e}$ definição de área de interesse. ${ }^{11 ; 12}$ Em recente discussão sobre o fomento da Medicina de Emergência nos Estados Unidos junto à agência financiadora estatal (National Health Institute), foi levantado o questionamento sobre qual seria a hipótese de pesquisa da área que a tornaria diferente e justificaria o investimento. Embora se trate de uma discussão em andamento, a definição que se consolida é que "o rápido diagnóstico e a intervenção precoce em enfermidades agudas ou em descompensações agudas de condições crônicas melhore o prognóstico dos pacientes atendidos". ${ }^{11 ; 12}$ Esta definição redireciona o foco tradicional de uma área de atuação baseada em sistemas ou órgãos, para o processo de atendimento ao paciente. Desta forma, passa-se a discutir a interação entre serviços pré-hospitalar, intra-hospitalar e 
pós-hospitalar, na definição de uma "cascata" de atendimento que tem sido motivo de redefinição de atendimento nos "bolsões" de influência no modelo flexineriano tradiconal vigente. ${ }^{13-16}$ Também deve ser discutida a sistemática de ensino e raciocínio nestas situações em comparação com modelos tradicionais. ${ }^{17}$ Por definição, portanto, a Medicina de Emergência passa a ter um caráter contestador e modificador de processo, voltado para o desfecho, discutindo itens como custo-efetividade e risco-benefício de modo muito mais precoce que outras áreas. Um exemplo cabal deste tipo de atuação voltada para o processo pode ser ilustrado pela própria iniciativa das sociedades americanas de emergência e de terapia intensiva discutirem formação conjunta de seus profissionais para garantir a "cascata". 18

Este artigo tem a finalidade de caracterizar a Medicina de Emergência na Faculdade de Medicina de Ribeirão Preto da Universidade de São Paulo (FMRP-USP). Ele irá resgatar o contexto histórico desta necessidade, contextualizar as condições atuais e apontar as providências para que esta meta seja efetivada. Não há como fugir, no entanto, de que o que ocorre na FMRP-USP reflete em muito a realidade de outras universidades brasileiras e, sem dúvida, a discussão aqui colocada pode ser de extrema utilidade.

\section{Aspectos Históricos}

Muito embora a recuperação do contexto enfrentado pela FMRP em termos de Medicina de Emergência seja complexa, ela provavelmente passou por etapas muito similares às documentadas no Brasil e no mundo. Em apresentação de um simpósio sobre Emergências Clínicas datado de 1982 na Revista Medicina, o Prof. Dr. Hélio Lourenço de Oliveira $^{19}$, então Chefe do Departamento de Clínica Médica, faz um resumo da evolução histórica da Emergência no Brasil: "Há uma geração, quando eram jovens os pais dos atuais jovens médicos, programas de residência médica foram iniciados nos principais hospitais do Rio e de São Paulo; neles se tornou possível o atendimento ininterrupto, aberto portanto às emergências. Começaram a existir os Pronto-Socorros nos hospitais-escola. Esta circunstância evidenciou que era geral a inexperiência e insegurança. As dúvidas, até completo desarvoramento, em que podiam ver-se jovens residentes diante de certos casos não eram, em geral, sanadas pelos docentes a quem recorriam". A descrição apresentada destaca alguns pontos, sendo o primeiro que a Medicina de Emergência se trata de especialidade que surge com a urbanização crescente e com a instituição de centros hospitalares de referência. ${ }^{1}$ Embora muito se discuta sobre a necessidade de hierarquização de sistemas no atendimento às emergências, isto é inerente ao seu surgimento. Um segundo aspecto que a descrição incorpora é a coragem de descrever a incapacidade de docentes especializados, acostumados à situações ambulatoriais ou de enfermarias comuns, de orientar residentes em situações de emergência. Finalmente, descreve o anseio da população médica em formação em adquirir o aprendizado de como lidar com estas situações.

A recuperação dos aspectos históricos para a demanda da individualização e implementação de disciplinas de emergências no contexto da FMRP-USP é tarefa hercúlea. No entanto, como a Unidade de Emergência se tornou o referencial físico dos problemas relativos à Emergência no decorrer da história da Instituição, isto permite que grande parte dos problemas discutidos seja rastreada através de processos administrativos que façam menção à Unidade. ${ }^{20}$

A implantação da Unidade de Emergência (UE) em 1982, com a mudança das atividades de ensino e assistência para o recém inaugurado HC Campus, foi motivada pela premência em se consolidar o atendimento de emergência para o ensino de alunos de graduação ${ }^{20}$, e a necessidade política de se estruturar o atendimento de emergência para a região de Ribeirão Preto. A atividade educacional neste primeiro momento de implantação "restringia-se ao necessário para oferecer oportunidades de treinamento a médicos residentes e aos internos que lá estagiavam. Um docente era encarregado de supervisionar essas atividades no período diurno, e um grupo de docentes, em rodízio, acompanhava os estagiários em suas atividades no período noturno", conforme detalhado em ofício do Prof. Dr. Ricardo Brandt de Oliveira para solicitação de claro docente para a Unidade datado de 3 de janeiro de 2003.

Em 1985, como documentado no Processo FMRP-USP 165/80 - Relatório da Comissão para estudo da posição da FMRP relativo ao aumento de leitos na Unidade de Emergência do HCFMRP, ${ }^{21}$ uma extensa análise do que se esperava da Unidade de Emergência foi realizada com vários pontos importantes podendo ser destacados. Em primeiro lugar, o anseio para o ensino de emergências 
era uma demanda antiga dos alunos de graduação em Medicina. Em segundo lugar, diversos Departamentos procuravam contemplar esta necessidade estabelecendo parcerias com a comunidade. Em terceiro lugar, o relatório deixa claro que "estas tentativas, no entanto, jamais conseguiram dissimular as nossas deficiências de ensino no setor específico da medicina de urgência".

Estas considerações deixam claro que a demanda pelo ensino de Emergência é antiga, antecedendo a fundação da Unidade de Emergência e o próprio relatório enfatiza que a FMRP, por ocasião da fundação da UE já “... não pode ignorar a crescente importância da medicina de urgência”. Um último e importante aspecto que deve ser enfatizado e que estava presente no referido processo é que "o atendimento médico na UE deve ser dirigido e supervisionado por docentes da FMRP, tanto pela sua vinculação ao ensino e pesquisa como pela necessidade de integração administrativa e funcional entre a UE e o HC Campus". Em que se pese esta recomendação, as décadas seguintes testemunhariam o afastamento progressivo entre as duas unidades, com estabelecimento de culturas distintas de atendimento e de acesso, em muito calcadas no tipo de paciente atendido. A atividade docente se mostrou incongruente em alguns aspectos e houve dificuldade de fixação e mesmo de coordenação de algumas áreas.

A importância das questões levantadas por aquele documento foi reconhecida pela Universidade de São Paulo que agraciou a FMRP com 11 dos 18 claros docentes solicitados para atuação na UE para os diversos Departamentos (Tabela 1). Aos Departamentos agraciados foi reforçada a autonomia de decidir sobre o modo de inserção dos docentes na UE e na estruturação do ensino de Emergência. No entanto, apesar de algumas iniciativas, os docentes contratados não chegaram a atuar na Unidade de Emergência.

A pressão pela Medicina de Emergência não se ateve, no entanto, à Unidade de Emergência. Em março de 1993, houve o início das atividades do Pronto Atendimento do Centro de Saúde Escola (PA-CSE) "Dr. Joel Domingos Machado" da Rua Cuiabá(22). Muito embora o CSE já fosse um serviço de extensão da FMRP-USP desde 1979, até então funcionava apenas como unidade básica de saúde com serviços ambulatoriais agendados. O fechamento temporário do Pronto Socorro Central de Ribeirão Preto forçou a Prefeitura Municipal a estabelecer postos de pronto- atendimento nas distritais de saúde e o CSE se tornou uma destas unidades, muito embora o convênio com a Prefeitura tenha sido formalizado apenas em 1999 e somente em renovações recentes do termo de compromisso foi incluída a participação da FAEPA (Fundação de Apoio ao Ensino, Pesquisa e Assistência). O Pronto Atendimento era um modelo novo, gerando controvérsia - "À medida que o serviço era implantado, percebiam-se as ambiguidades e expectativas diversas da clientela, na equipe do PA e nas equipes das outras unidades de saúde. Mesmo os técnicos da secretaria divergiam quanto ao que devia ser, ou não, atribuição do PA"(22). Os principais problemas identificados foram: 1) inexistência da integração do modelo de Pronto Atendimento com os demais serviços da rede para garantir o atendimento continuado; e a 2) inabilidade dos profissionais transferidos para o PA em lidar com situações de emergência, o que gerou refratariedade ao modelo e grande giro de profissionais. A demanda por profissionais capacitados em Medicina de Emergência já existia e era enfrentada, mas como voltaria a ocorrer repetidamente no futuro, a área de atendimento de emergência não se tornou uma prioridade dentre as múltiplas demandas existentes na FMRP.

Retornando o foco para a UE, o insucesso da iniciativa de inserção docente fica claro num documento desenvolvido uma década depois do relatado anteriormente, datado de outubro de 1997, intitulado Corpo docente da Faculdade de Medicina de Ribeirão Preto-USP: Necessidades atuais e política de expansão(23). Este documento foi elaborado por uma comissão nomeada pelo Conselho Técnico-Administrativo (CTA), sendo discutido e aprovado em sua 97ª Sessão Ordinária de 15 de agosto de 1997.

Esse documento explicita a opinião do corpo docente em relação ao "ensino da Medicina de Urgência ainda é um capítulo não satisfatoriamente resolvido no âmbito da FMRP. Por um lado existe toda uma infraestrutura hospitalar especializada para tal que, se não é ainda a ideal, vem sendo progressivamente melhorada ao longo dos anos. Entretanto, a atual sistemática de designação de docentes pelas Clínicas da FMRP mantém-no deficitário, além de colocar em evidência alguns aspectos até agora controvertidos entre os próprios docentes, em vista da exposição de estudantes profissionalmente imaturos a um Sistema Unificado de Saúde ainda desorganizado. Ninguém nega a importância do local para fins de ensino, 
mas, ao que tudo indica, será necessário organizar ali um Departamento de Medicina de Urgência ou um Núcleo Interdepartamental de Medicina de Urgência". Após discorrer sobre todas as outras deficiências do corpo docente na FMRP-USP, o documento finaliza com recomendações de ajuste de corpo docente e enfatiza que seria necessária a contratação de 12 docentes para a Unidade de Emergência. ${ }^{23}$ Estes claros não foram concedidos e não houve destinação para Departamentos específicos. (Tabela 1)

Embora o referido documento não seja explícito quanto ao motivo do insucesso da implementação dos 11 claros adquiridos em 1985 para atuação na UE, pode-se depreender alguns aspectos que sem dúvida contribuíram para isto. Em primeiro lugar, a política de contenção de gastos da USP penalizou sobremaneira a FMRP, provavelmente forçando que seu corpo docente sobrecarregado priorizasse áreas já estabelecidas em detrimento de implantação de novas áreas. ${ }^{23} \mathrm{Um}$ segundo aspecto que provavelmente teve influência foi a situação desfavorável de trabalho na UE naquela ocasião, que não propiciava as condições mínimas de pesquisa que mantivessem o interesse do corpo docente. ${ }^{20 ; 24}$ Também é notório que, a solicitação de claros não foi pautada num planejamento de inserção destes docentes e nem associado a reformas curriculares que favorecessem a consolidação do modelo. Ao respeitar a autonomia departamental, a Universidade abriu mão do seu caráter fiscalizador para que o processo fosse implementado, e os docentes foram desviados para outras atividades no HC-Campus.

Posteriormente a estas manifestações institucionais da FMRP, os esforços foram voltados para outras áreas de atuação. Nos anos que se seguiram a

\section{Tabela 1}

Distribuição de claros docentes para Medicina de Emergência na FMRP-USP de acordo com o ano de solicitação e Departamento/Disciplina agraciado e número de docentes em atuação em 2010 na Unidade de Emergência de acordo com o tipo de atuação e Departamento/Disciplina

\begin{tabular}{|c|c|c|c|c|c|c|c|c|c|c|c|c|c|}
\hline \multirow[b]{2}{*}{ Área } & \multicolumn{3}{|c|}{1986} & \multicolumn{2}{|c|}{1997} & \multicolumn{3}{|c|}{2008} & \multicolumn{2}{|l|}{ Total } & \multicolumn{3}{|c|}{2010} \\
\hline & $\begin{array}{c}\text { Solici- } \\
\text { tado }\end{array}$ & $\begin{array}{l}\text { Pro- } \\
\text { vido }\end{array}$ & $\begin{array}{l}\text { Empos- } \\
\text { sado } \\
\text { na UE }\end{array}$ & $\begin{array}{l}\text { Solici- } \\
\text { tado }\end{array}$ & $\begin{array}{l}\text { Pro- } \\
\text { vido }\end{array}$ & $\begin{array}{c}\text { Solici- } \\
\text { tado }\end{array}$ & $\begin{array}{l}\text { Pr } \\
\text { USP }\end{array}$ & $\begin{array}{l}\text { vido } \\
\text { FAEPA }\end{array}$ & $\begin{array}{l}\text { Solici- } \\
\text { tado }\end{array}$ & $\begin{array}{l}\text { Pro- } \\
\text { vido }\end{array}$ & $\begin{array}{l}\text { Inte- } \\
\text { gral }\end{array}$ & $\begin{array}{l}\text { Par- } \\
\text { cial }\end{array}$ & $\begin{array}{c}\text { Total } \\
\text { em } \\
\text { atuação }\end{array}$ \\
\hline $\begin{array}{l}\text { Clínica } \\
\text { Médica }\end{array}$ & 3 & 1 & 0 & & & 1 & 1 & 0 & & 2 & 2 & 0 & 2 \\
\hline Pediatria & 3 & 3 & 1 & & & 1 & 0 & 1 & & 4 & 1 & 3 & 4 \\
\hline Neurologia & a 2 & 1 & 0 & & & 1 & 1 & 0 & & 2 & 1 & 0 & 1 \\
\hline Cirurgia & 2 & 1 & 0 & & & 1 & 1 & 0 & & 2 & 1 & 0 & 1 \\
\hline Ortopedia & 2 & 1 & 0 & & & 0 & 0 & 0 & & 1 & 0 & 1 & 1 \\
\hline $\begin{array}{l}\text { Ginecolo- } \\
\text { gia e Obs- } \\
\text { tetrícia }\end{array}$ & 2 & 1 & 0 & & & 1 & 0 & 1 & & 2 & 0 & 2 & 2 \\
\hline Psiquiatria & 1 & 0 & 0 & & & 0 & 0 & 0 & & 0 & 0 & 1 & 1 \\
\hline $\begin{array}{l}\text { Oftalmo- } \\
\text { logia }\end{array}$ & 1 & 1 & 0 & & & 0 & 0 & 0 & & 1 & 0 & 0 & 0 \\
\hline $\begin{array}{l}\text { Otorrinola- } \\
\text { ringologia }\end{array}$ & -1 & 1 & 0 & & & 0 & 0 & 0 & & 1 & 0 & 0 & 0 \\
\hline Anestesia & 1 & 1 & 0 & & & 0 & 0 & 0 & & 1 & 0 & 0 & 0 \\
\hline Total & 18 & 11 & 1 & 12 & 0 & 5 & 3 & 2 & 35 & 16 & 5 & 7 & 12 \\
\hline
\end{tabular}


este relatório de 1997, a implantação dos cursos de Fisioterapia, Terapia Ocupacional, Nutrição, Fonoaudiologia e Informática Biomédica, a estruturação do Centro de Saúde-Escola da Cuiabá e os esforços para se ampliar outros espaços na comunidade para o ensino de medicina primária e secundária, como o Hospital Estadual Regional, a incorporação da Mater, e os Núcleos de Saúde da Família desviaram os esforços para outras áreas. Neste ponto deve ser ressaltada uma característica constante de vários processos de ampliação da FMRP-USP, nos quais a existência da Unidade de Emergência é considerada como estrutura suficiente para garantir atendimento aos pacientes que deverão ser abordados nestes novos serviços ou como campo de treinamento adequado para inserção de alunos dos novos cursos criados. Não é incluído, dentro do planejamento destas ampliações, investimento de estrutura física ou de pessoal capacitado a ser agregado à UE para cumprir esta missão. Também não é previsto aumento da capacidade de atendimento de emergência em outras esferas que não a UE.

O desvio de esforços para outras áreas que não a Medicina de Emergência provavelmente teve influências políticas das diversas áreas de atuação da FMRP-USP, mas sem dúvida alguma, foi influenciado também pela forte crise que a Unidade de Emergência enfrentou no período que se estendeu de 1993 a 1998. ${ }^{20 ; 24}$ Neste período, a epidemia de Síndrome da Imuno-Deficiência Adquirida e a completa desestruturação do Sistema Único de Saúde transformaram a Unidade de Emergência em sinônimo de desorganização e descuido, o que fez com que diversos Departamentos da FMRP considerassem inclusive a sua retirada da Unidade, sugerindo que ela fosse devolvida ao Estado para providências. Nesse contexto, é compreensível que a inserção docente tenha sido, mais uma vez, desencorajada e mesmo questionada, pois as atividades de ensino e pesquisa estavam muito ameaçadas ou eram inexistentes.

Em 1999, com a destinação de verbas do ReforSUS e da FAEPA para a Unidade, iniciava-se o processo de recuperação. ${ }^{24} \mathrm{~A}$ Unidade de Emergência foi reinserida no SUS com o papel de referência terciária. A instalação de um Sistema de Regulação Médica na DRS XIII modificou drasticamente o perfil dos pacientes encaminhados para a Unidade, que passou a ser modelo nacional de cuidado aos pacientes críticos. Este processo de modificação de paradigma ainda está em curso, mas já propicia condições para que o ensino de graduação e pós-graduação sen- so latu (residência médica) seja realizado na Unidade com carga horária considerável. Estas condições reavivaram os anseios antigos da FMRP de estabelecer ensino e pesquisa de qualidade em Emergência.

$\mathrm{O}$ aditivo de convênio com a Prefeitura Municipal de Ribeirão Preto para a "operacionalização do Serviço de Pronto Atendimento da Unidade Básica Distrital de Saúde do Sumarezinho, Centro de Saúde Escola da FMRP-USP, serviço que funciona ininterruptamente nas 24 horas diárias" foi firmado também em 1999. Desde então, a FMRP-USP tem investido consideráveis recursos na estruturação do Pronto Atendimento para garantir as obrigações contratuais e enfrentou severas críticas por parte da imprensa leiga. Deve ser contextualizado que nos últimos anos houve um enorme avanço estrutural nas condições de atendimento, mas ainda impera dificuldade de estabelecimento do profissional de emergência, que não se fixa em virtude do excesso de trabalho, baixos salários e inexistência de um plano de carreira. Geralmente, estes profissionais são de diversas especialidades e exercem a atividade no PA como um primeiro emprego, até que se fixem na especialidade de origem.

Não se pode deixar de mencionar, no entanto, o empenho de poucos docentes que ao longo de vários anos foram baluartes da resistência em se manter na UE e a Medicina de Emergência na FMRP-USP, mantendo e implementando melhorias na estrutura. Deve ser também enfatizado, que a maioria destes docentes não foi contratada com vagas agraciadas à FMRPUSP oriundas dos esforços de 1985 (Tabela 1). Graças ao esforço destes profissionais, gradativamente e de forma isolada, alguns Departamentos da FMRPUSP voltaram a ocupar espaço na UE para o ensino de médicos residentes e alunos. Houve iniciativas de reposição de vagas docentes. Algumas áreas, pela interrelação forte com a Medicina de Emergência foram se fixando na UE e mais recentemente no PACSE. Esta organização incipiente resultou na inserção curricular de disciplina voltada para a Cirurgia do Trauma, em 1994, que evolui para o ensino de Primeiros Socorros (RCG 0221 - Primeiros Socorros e Atendimento Pré-Hospitalar), em 2003. Foram instituídos cursos de treinamento em emergência para situações específicas de trauma, suporte básico de vida e suporte avançado de vida em cardiologia e pediatria. ${ }^{25 ; 26}$ A organização destes docentes culminou com solicitação à diretoria da FMRP para que a Emergência voltasse a ser incluída como prioridade institucio- 
nal. O processo foi então formalizado com a Portaria D. $\mathrm{N}^{\mathrm{o}} 23 / 08$, de 10 de março de 2008 que estabeleceu uma comissão para definir um projeto institucional para as emergências no contexto da FMRP (Processo USP 07.1492.17.3). O relatório desta comissão foi entregue em 19 de maio de 2008. Este relatório deu origem ao processo em vigência que se constitui numa nova tentativa de sedimentar a Medicina de Emergência na FMRP-USP e mantém a Medicina de Emergência como meta institucional desde então. Através dele, a experiência histórica revista até o momento (Figura 1) foi contextualizada e a proposta visa corrigir os erros apontados.

\section{A estruturação da medicina de emergência na FMRP-USP no sécu- lo 21}

Em resumo, a Medicina de Emergência, embora ainda não considerada como tal no Brasil, é uma especialidade médica que objetiva o processo de atendimento ágil e eficaz em situações de agravo agudo à saúde das pessoas, pressupondo hierarquização de estrutura. Está extremamente relacionada com outras áreas de atuação e deve garantir um fluxo ("cascata") de atendimento tanto no aspecto estrutural (pré, intra e pós-hospitalar) como no aspecto inter-disciplinar. Trata-se de uma especialidade que funciona como um indicador precoce da atuação do sistema de saúde, apontando suas falhas e necessidades de melhoria. Tem sido excluída de projetos de expansão de outras áreas de atuação, sendo erroneamente interpretado que organizar a estrutura seja suficiente, e que a formação em emergência é garantida pela própria formação em medicina.

Várias iniciativas têm buscado modificar este ponto de vista, sendo que o foco atual está centrado nas sociedades médicas, pela própria demanda de mercado. Em que se pese a necessidade desta organização no curto prazo, a formação de profissionais ca-

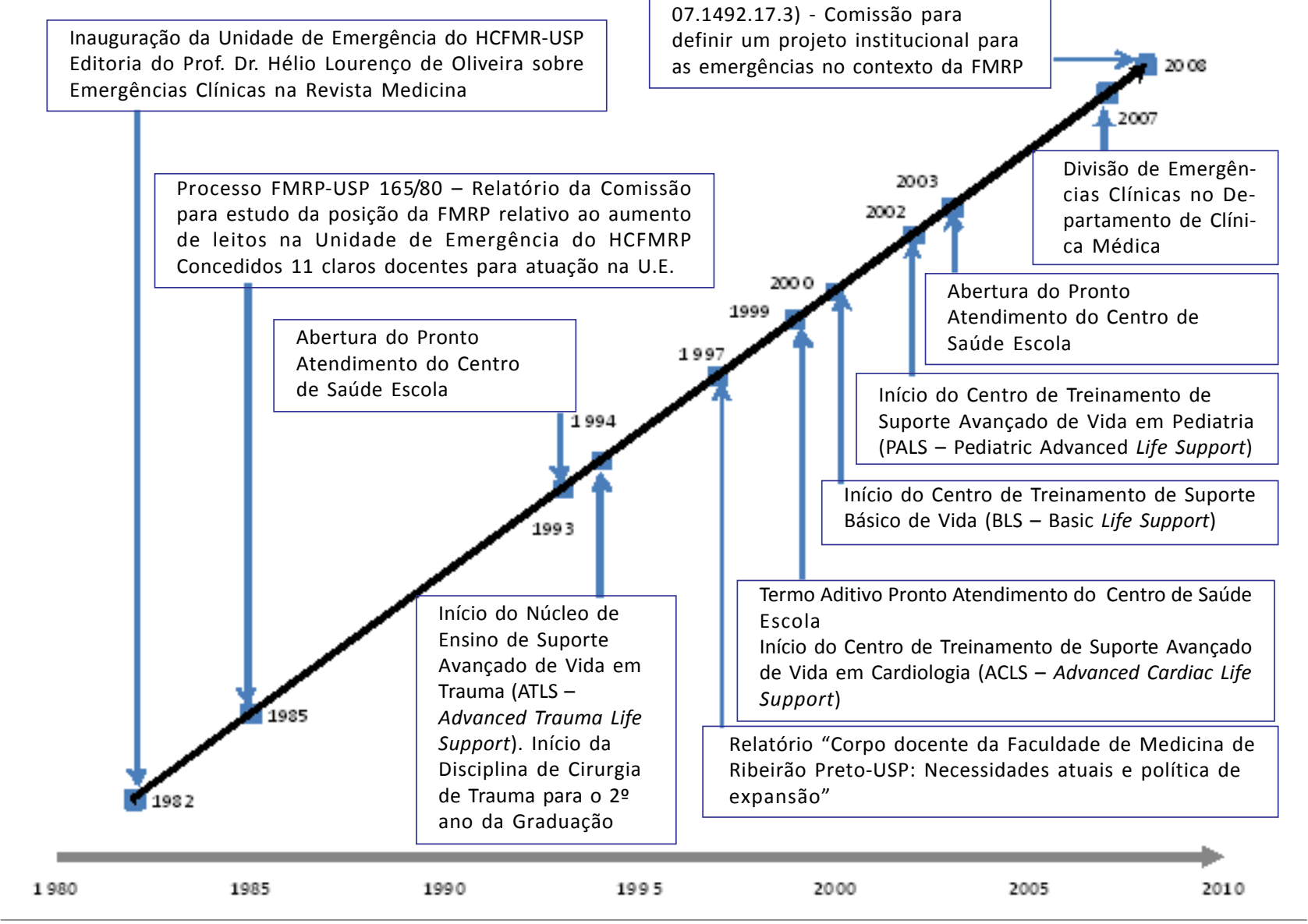

Figura 1: Aspectos históricos de relevância para a Medicina de Emergência na FMRP-USP. 
pacitados em emergência deve se iniciar na graduação, sendo esta a única iniciativa que irá garantir a modificação cultural necessária no médio e no longo prazo.

Neste contexto, a proposta vigente na FMRPUSP busca respeitar as necessidades da especialidade, está embasada na infra-estrutura construída nas últimas décadas como locais de ensino e se adapta ao modelo educativo proposto pela Comissão de Graduação na forma de eixo longitudinal de ensino ao longo dos seis anos de graduação em Medicina (Figura 2). Este processo foi aprovado pela Comissão de Graduação $\left(720^{a}\right.$ Reunião Ordinária) e pela Congregação (743 $3^{\text {a }}$ Reunião Ordinária) da FMRP-USP para ser incluído na atual reestruturação do currículo do curso médico e está em fase de implantação. O suporte institucional foi conseguido, tendo sido fornecidos 3 claros docentes USP e dois claros FAEPA (Tabela 1), dos quais 4 docentes já foram contratados. Como a estrutura da Universidade de São Paulo requer vinculação departamental, as vagas foram distribuídas conforme descrito na Tabela 1, mas estão sendo implementadas estratégias para se manter a adesão dos docentes contratados, como o estabelecimento do Centro Integrado de Emergências em Saúde (CIES), aprovado pela Congregação na sua $761^{\mathrm{a}}$ Reunião Ordinária, e atualmente em tramitação na Consultoria Jurídica da USP. O projeto deixa claro também que, à medida que se desenvolva o eixo proposto, haverá a necessidade de incorporação de docentes de outras especialidades, de acordo com a epidemiologia das situações de emergência.

A primeira etapa do eixo está constituída pelo curso de Primeiros Socorros já instaurado desde 2003 (Figura 1). Este curso anteriormente era ministrado no segundo ano do curso médico, tendo sido realocado para o primeiro ano na reforma curricular atual. Neste momento, são fornecidos princípios básicos de como proceder numa situação de emergência e sobre a estrutura do sistema de saúde. O foco é o mesmo fornecido ao leigo, pois se trata de alunos do primeiro ano do curso médico. Este curso foi realizado por muitos anos com recursos dos cursos de suporte de vida e, recentemente, por iniciativa da Comissão de Graduação e instalação do Laboratório de Habilidades, foram adquiridos manequins para as práticas de habilidades. $^{25,26,27}$

A segunda etapa do eixo está prevista para se iniciar em 2011, sendo incluída como um curso de 30 horas no quarto ano do currículo do curso médico (Fi- gura 2). O curso será constituído de estações de habilidade e simulações de situações de emergência, sendo fornecido no Laboratório de Habilidades. Os objetivos nesta etapa visam fornecer fundamentos de procedimentos a serem realizados em situações de emergência. Foram derivados da percepção de que os alunos do currículo anterior, ao atingir os estágios de aplicação no internato, não tinham base teórica ou prática consolidada dos procedimentos mais comuns, limitando sua oportunidade de prática. A utilização de recursos de Simulação irá permitir a compreensão da prática de procedimentos como uso de desfibriladores, procedimentos de punção venosa profunda, etc. Esta delimitação segue a tendência presente não só em Medicina de Emergência, mas também em diversas outras especialidades de permitir que o aluno pratique primeiro em modelos simulados para evitar exposição desnecessária de pacientes. ${ }^{28}$ Há reconhecimento da FMRP-USP da necessidade deste Laboratório, sendo prevista a expansão de sua área física e maiores recursos orçamentários para a compra de equipamentos. Também foi estabelecida pela Comissão de Graduação uma comissão de docentes para gerir o Laboratório e orientar a inserção de todas as áreas que tiverem interesse em utilizar a técnica. Finalmente, há iniciativas de capacitação docente que já foram implementadas e que deverão ser expandidas.

A terceira etapa deverá ser inserida no internato do curso médico a partir de 2012 (quinto ano - Figura 2). Ela será desenvolvida no PA-CSE, visando fornecer ao aluno quatro princípios básicos. Do ponto de vista técnico, permitirá o contato com situações de emergência de menor complexidade, pois o PA-CSE é um modelo de pronto socorro de "porta aberta", ou seja, que atende demanda espontânea da população. A necessidade deste ambiente de ensino foi observada pelo aumento da complexidade dos casos encaminhados à UE com a hierarquização do sistema de saúde, quando esta teve seu acesso restrito a casos referenciados (modelo de "Regulação Médica"). Para que o aluno seja exposto a casos de menor complexidade que são os mais prevalentes em situações de emergência, ele deverá sair do ambiente da UE. Um segundo aspecto diz respeito à estrutura de sistema, sendo que o aluno deverá aprender técnicas de priorização de atendimento. No momento, encontra-se em implantação um projeto de estratificação de risco para determinar os pacientes que devem ser atendidos prioritariamente em função do risco iminente de vida ou perda de função. Este é um recurso que está se 


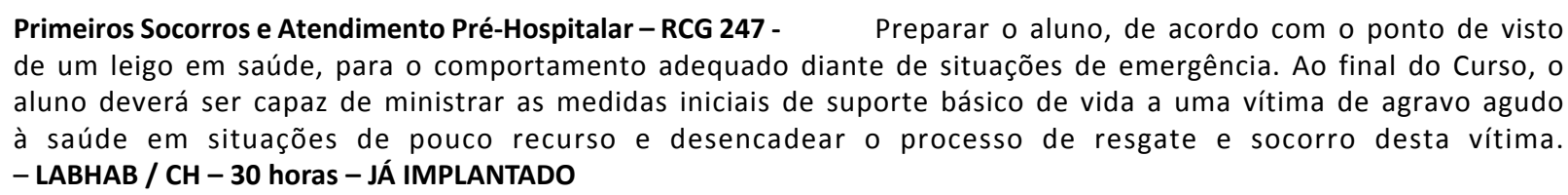

Figura 2: Eixo temático em Medicina de Emergência aprovado pela Comissão de Graduação da FMRP-USP na $720^{\underline{a}}$ Reunião Ordinária de 29 de julho de 2008

expandindo nas salas de emergência não referenciadas do país e que necessariamente fará parte da vida profissional dos egressos da FMRP-USP. O terceiro objetivo a ser atingido é a conciliação dos dois anteriores, sendo esperado do aluno que ele seja capaz de identificar casos que não podem ser resolvidos nesta instância e ative o sistema para transferência do paciente. Finalmente, será buscada a integração com as outras instâncias do sistema de saúde, especialmente os núcleos de Medicina de Família e as Unidades Básicas de Saúde, pois também será esperado que o aluno possa identificar casos que não devam ser atendidos no pronto socorro e possam ser seguramente encaminhados para estas instâncias, ou mesmo que possam ser contra-referenciados após a intervenção pontual na emergência. Isto irá garantir a visão holística do sistema e a recuperação de conceitos já aplicados no eixo de Atenção à Saúde da Comunidade, também parte do currículo da FMRP-USP.

Esta terceira etapa do eixo ainda está em planejamento, restando ainda a discussão sobre a carga horária a ser alocada e o formato a ser desenvolvido. No entanto, pela necessidade de estruturação do PACSE, atendendo ao pedido da Diretoria da FMRPUSP e da Coordenadoria do CSE, já se iniciaram atividades neste ambiente. Cinco pós-graduandos fomentados pela Diretoria da FMRP-USP já se encontram supervisionando o atendimento de alunos do quinto ano 
médico do currículo atual. Este projeto visa documentar os pontos fortes e a serem melhorados para que se estabeleça o ensino de graduação em emergência. Neste sentido, já foram identificadas medidas Administrativas e Acadêmicas (Figura 3) que serão necessárias e foi traçado um plano de metas a ser atingido. Como exemplos, serão buscadas estratégias para aumentar o contingente de médicos para supervisão, sua qualificação e fixação no setor, a inserção coordenada de médicos residentes de diversos programas e a solicitação da criação da vaga do estágio em Medicina de Emergência, mesmo que ainda considerado como "área de atuação" pelo Conselho Nacional de Residência Médica” e pela Associação Médica Brasileira (já solicitada à Comissão de Residência Médica do HCFMRP-USP e aguardando visita de credenciamento).

Finalmente, a quarta etapa do eixo deverá ser alocada no sexto ano médico, desenvolvendo-se na Unidade de Emergência e prevista para 2013. Como parte do internato, terá como objetivo a prática supervisionada num ambiente de referência terciária, no qual o aluno agora será exposto a casos de maior gravidade. Será necessário reestruturar o atendimento de emergência atualmente vigente na UE, pois apesar da excelente estrutura física, o proces- so ainda é fragmentado em diversas áreas, dificultando a compreensão integral da abordagem do paciente de emergência.

Muito embora ainda não se tenha garantias do sucesso de sua implementação, o presente projeto tem maiores chances de ser bem sucedido quando comparados às tentativas anteriores. Conta com melhores condições de estrutura física para inserção do aluno, com o apoio institucional e foi inserido na estrutura curricular. Além disso, está sendo desenvolvido num momento histórico diferente, no qual a pressão social para o desenvolvimento da Medicina de Emergência é maior.

No entanto, a fiscalização contínua do projeto deve ser buscada e a FMRP-USP deve assumir sua responsabilidade no processo. Índices de acompanhamento devem ser estabelecidos e fiscalizados periodicamente, evitando-se desvios que já ocorreram no passado, principalmente no concernente ao efetivo docente adquirido. Estas metas devem levar em consideração principalmente o ensino médico e terem em vista o todo do projeto, ao invés de fiscalizar apenas o desempenho docente através de índices voltados para a produção acadêmica em pesquisa. Quando se fala em fiscalização, não se deve ter em mente apenas a Diretoria ou a Congregação da FMRP-USP, mas tam-

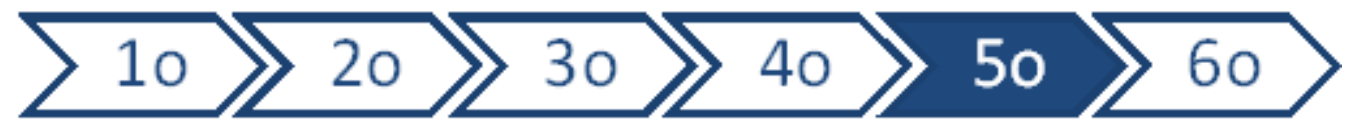

\begin{tabular}{lll} 
Prazo & Assistencial & Acadêmico \\
\hline Curto (2010) & 1. Inserção do quarto médico no período da & 1. Inserção docente no CSE \\
& manhã e da tarde & 2. Preparo de propostas de Residência para os \\
& diversos programas
\end{tabular}

Médio (2011) $\begin{aligned} & \text { 1. Inserção do quarto médico no período da noite } \\ & \text { (elevado movimento até 23horas) } \\ & \text { 2. Início das atividades de Acolhimento e } \\ & \text { Classificação de Risco (ACR) }\end{aligned}$

Longo (2012) 1. Sedimentação progressiva do ACR 2. Interação com Medicina de Família
1. Inserção do médico com perfil acadêmico (gradativa substituição do pós-graduando)

2. Medicina de Emergência

3. Participação de residentes de outros programas

4. Estruturação do curso do quinto ano médico

1. Início das atividades do quinto ano de medicina

Figura 3: Providências de curto, médio e longo prazo planejadas do ponto de vista assistencial e acadêmico para a inserção da terceira etapa do eixo de Medicina de Emergência. 
bém o seu corpo discente, que deve zelar e contribuir para que as metas aqui descritas sejam atingidas.

A Medicina de Emergência está deixando de ser uma necessidade para se tornar uma realidade. Cabe à FMRP-USP decidir se esta proposta será apenas mais uma tentativa como as anteriores ou se ocupará o papel pioneiro de inovação e seriedade na área, a exemplo do que ocorre em tantos outros setores da Faculdade.

\section{Agradecimentos}

Os autores são gratos aos esforços das Sras. Renata Aparecida Terra Cazarotti, Assistente Técnica Acadêmica da FMRP-USP; Deocelia Bassotelli Jardim, Assistente da Diretoria da FMRP-USP, e Cleide Filipini, ex-Assistente Acadêmica da FMRPUSP, pela enorme contribuição na localização de documentos que substanciaram este artigo.

\begin{abstract}
Emergency Medicine, although not considered as such in Brazil, is a medical specialty that aims agile and effective action in situations of acute injury to the health of people, assuming a hierarchical structure. Is extremely related to other areas and should ensure a flow ("waterfall") service in both the structural aspect (pre, intra and post-hospital) and inter-disciplinary aspect. Several initiatives have sought to insert it in the Brazilian reality, and the current focus is centered on the medical societies due to market demand. In spite the need for this organization in the short term, the training of professionals trained in emergency should start in their own graduation, which is the only initiative that will ensure the necessary cultural change in the medium and long term. The Faculty of Medicine of Ribeirao Preto, University of São Paulo (FMRP) has had several attempts at this integration throughout its history, which is rescued in this article. Currently, there is a new project that proposes its inclusion in a longitudinal curriculum that seeks to meet the needs of the specialty is based on the infrastructure available to local education and adapts to the educational model proposed by the Commission of Graduation. The proposal is summarized, pointing out its principles and goals for each stage and reinforcing the need for institutional support and oversight to its success.
\end{abstract}

Key-words: Emergency Medicine. Education, Medical. History of Medicine.

\section{Referênclas Blbllográflcas}

1. Zink BJ. Anyone, anything, anytime - a history of emergency medicine. 1st ed. Philadelphia: Mosby Elsevier; 2006.

2. Thomas TL. Developing and implementing emergency medicine programs globally. Emerg Med Clin North Am 2005 Feb;23(1):177-97.

3. Pazin-Filho A, Peitz P, Pianta T, Carson KA, Russell SD, Boulware LE, et al. Heart failure disease management program experience in 4,545 heart failure admissions to a community hospital. Am Heart J 2009 Sep;158(3):459-66.

4. Razzak JA, Kellerman AL. Emergency medical care in developing countries: is it worthwhile? Bulletin of the World Health Organization 2002;80(11):900-5.

5. Keyes C, Rodriguez-Gomez G, Quesada-Rodriguez D, Waller J. The Costa Rican emergency medicine residency: design and implementation of a new specialty training program in Central America. Ann Emerg Med 1999;34(6):790-5.

6. Kirsch TD. Emergency medicine around the world. Ann Emerg Med 1999;32(2):237-8.

7. Tannebaum RD, Arnold JL, De Negri Filho A, Spadoni VS. Emergency medicine in Southern Brazil. Ann Emerg Med 2001;37(2):223-8.
8. Timerman S, Gonzalez MM, Zaroni AC, Ramires JA. Emergency medical services: Brazil. Resuscitation 2006 Sep;70(3):356-9.

9. CREMESP. Especialidades médicas no Estado de São Paulo. CREMESP; 2008.

10. Lorin S, Heffner J, Carson S. Attitudes and perceptions of internal medicine residents regarding pulmonary and critical care subspecialty training. Chest 2005 Feb;127(2):630-6.

11. Courtney DM, Neumar RW, Venkatesh AK, Kaji AH, Cairns CB, Lavonas $\mathrm{E}$, et al. Unique characteristics of emergency care research: scope, populations, and infrastructure. Acad Emerg Med 2009 Oct;16(10):990-4.

12. Neumar RW. The Zerhouni challenge: defining the fundamental hypothesis of emergency care research. Ann Emerg Med 2007 May;49(5):696-7.

13. Cairns CB, Garrison HG, Hedges JR, Schriger DL, Valenzuela TD. Development of new methods to assess the outcomes of emergency care. Acad Emerg Med 1998 Feb;5(2):157-61.

14. Chang SY, Multz AS, Hall JB. Critical care organization. Crit Care Clin 2005 Jan;21(1):43-53, viii.

15. Ghosh R, Pepe P. The critical care cascade: a systems approach. Curr Opin Crit Care 2009 Aug;15(4):279-83. 
16. Hollander JE, Gaulton GN, Courtney DM, Lewis RJ, Lowe RA, Becker $\mathrm{MO}$, et al. Facilitating emergency care research networks: integration into the Clinical Translational and Science Award (CTSA) infrastructure. Acad Emerg Med 2009 Oct; 16(10):1005-9.

17. Neves FF, Pazin-Filho A. Raciocínio clínico na sala de urgência. Medicina (Ribeirão Preto) 2008;41(3):335-42.

18. Huang DT, Osborn TM, Gunnerson KJ, Gunn SR, Trzeciak S, Kimball E, et al. Critical care medicine training and certification for emergency physicians. Crit Care Med 2005 Sep;33(9):2104-9.

19. Oliveira HL. Simpósio Medicina de urgência - apresentação. Medicina (Ribeirão Preto) 1982;15(Supl.).

20. Santos JS. Da fundação do Hospital das Clínicas à criação da Unidade de Emergência e sua transformação em modelo nacional de atenção hospitalar às urgências. Medicina, Ribeirão Preto 2002;35(3):403-18.

21. Sader AA. Relatório da "Comissão para estudos da posição da FMRP relativa ao aumento de leitos na Unidade de Emergência". 1985. Report No.: 165/80.

22. Brito F, Marcellino I, Forster A, Simões B. Pronto-atendimento de adultos em serviço de saúde universitário: um estudo de avaliação. RAP 1998;32(3):47-64.
23. Oliveira JAM, Sakamato AC, Lodi WRN. Corpo docente da Faculdade de Medicina de Ribeirão Preto - USP: necessidades atuais e política de expansão. 1997. Report No.: 97.1.1219.17.9.

24. Santos J, Scarpelini S, Lopes S, Ferraz C, Dallora MELV, Sá MFS. Avaliação do modelo de organização da unidade de emergência do HCFMRP-USP, adotando, como referência, as políticas nacionais de atenção às urgências e humanização. Medicina, Ribeirão Preto 2003;36(2/4):498-515.

25. Pazin-Filho A, Schmidt A, Filipini C, Castro RBP, Rosa RM, Rosa MAF, et al. Simulação de pacientes - Cursos de suporte de vida - ACLS, BLS e PALS na FMRP-USP. Medicina (Ribeirão Preto) 2007;40(2):204-12.

26. Scarpelini S, Andrade JI. Simulação de pacientes - Cursos de suporte de vida - Suporte de vida avançado no trauma. Medicina (Ribeirão Preto) 2007;40(2):199-293.

27. Troncon LEA, Maffei CML. A incorporação de recursos de simulação no curso de graduação em medicina da Faculdade de Medicina de Ribeirão Preto - USP. Medicina (Ribeirão Preto) 2007;40(2):153-61.

28. Pazin-Filho A, Scarpelini S. Simulação: definição. Medicina (Ribeirão Preto) 2007;40(2):162-6. 